\title{
Correlative Electron Tomography and Elemental Microanalysis in Biology
}

\author{
M. Marko, ${ }^{*}$ T.W. Wagenknecht,* F. Ichas, ${ }^{* *}$ P. Bernardi, ${ }^{* * *}$ and C.A. Mannella*
}

*Resource for Visualization of Biological Complexity, Wadsworth Center, Empire State Plaza, Albany, NY 12201

**Institut Européen de Chimie et Biologie, 146 rue Léo Saignat, F-33076 Bordeaux, France

***Department of Biomedical Sciences, University of Padova, Viale Giuseppe Colombo 3, I-35131

Padova, Italy

Recently, EFTEM [1] and STEM [2,3] have joined conventional TEM as imaging modes for electron tomography. EFTEM tomography can be extended from zero loss imaging to low-loss and core-loss imaging for elemental mapping. Tomography in STEM mode is compatible with $\mathrm{x}$-ray and EELS spectrum imaging. Acquisition of an elemental map is more time consuming than recording a conventional image, thus an extensive tilt-series of elemental maps is often not practical. However, a limited tilt series, a stereo pair, or even a 2-D elemental map can be used to correlate elemental composition with the 3-D structure obtained from a previously recorded tomogram.

We have recently implemented electron tomography of frozen-hydrated tissue sections [4]. The advantage of this technique is that no chemical fixation, dehydration or staining is required, and diffusible elements are retained in place. However, the electron dose needed for even a single 2-D elemental map usually far exceeds that allowable for tomography of frozen-hydrated sections (5000 $\mathrm{e}^{-} / \mathrm{nm}^{2}$ ). For biological microanalysis, a standard method involves freeze-drying such sections in the EM $[5,6]$. Thus, for correlative work, a tomographic reconstruction made before freeze-drying can be compared with elemental maps acquired after drying. We are also applying this method in our work with frozen-hydrated suspensions of isolated organelles [7,8]. Since frozen-hydrated material is subject to collapse on freeze-drying, correlation with a 2-D elemental map may be sufficient.

Sections of conventional plastic-embedded specimens can also be used for microanalysis. An example of microanalysis work that should be compatible with tomography is EFTEM mapping of protein and nucleic acid in the nucleus using phosphorus and nitrogen imaging [9]. These elements are plentiful enough for a reasonable acquisition time per tilt image. Other examples might include identification of foreign material such as metals in tissue. Diffusible elements such as $\mathrm{Na}^{+}, \mathrm{K}^{+}$, and $\mathrm{Ca}^{++}$can be studied using plastic sections, although with less than ideal ultrastructural preservation, by the use of pyroantimonate precipitation [10] or anhydrous processing [11]. An advantage of using plastic sections is that there is no specimen collapse between tomography and microanalysis.

We have been investigating localization of $\mathrm{Ca}^{++}$in mitochondria and skeletal muscle triad junctions. In isolated mitochondria loaded with excess calcium, we find $\mathrm{Ca}$ in the matrix granules (Fig. 1). The mitochondrial work shown here was done using plastic sections [11], and will be compared with future work using frozen-hydrated specimens. We have also been studying isolated (but still functional) skeletal muscle triad junctions using frozen-hydrated preparation and cryo-electron tomography. We see densities in the sarcoplasmic reticulum (SR) that likely represent the protein calsequestrin [8]. In initial experiments (Fig. 2) Ca was barely detectable in SR vesicles, but we are hopeful that technical improvements will allow us to map distribution of $\mathrm{Ca}^{++}$within a vesicle.

References

[1] G. Moebus and B.J. Inkson, Proc. ICEM-15 Durban, SA 3 (2002) 375.

[2] M. Weyland et al., J. Phys. Chem. B. 105 (2001) 7882. 
[3] U. Zeise, et al., J. Struct. Biol. 139 (2002) 58.

[4] C.-E. Hsieh et al., J. Struct Biol. 138 (2002) 63.

[5] L. Edelman, L., Scanning Microsc. Suppl. 8 (1994) 67.

[6] S. Shi et al., Microsc. Res. Tech. 33 (1996) 241.

[7] C.A. Mannella et al., IUMB Life 52 (2001) 93.

[8] T.W. Wagenknecht et al., Biophys. J. 83 (2002) 2491.

[9] M.J. Hendzel et al., Mol. Biol. Cell 10 (1999) 2051.

[10] R.D. Happel and J.A.V. Simson, J. Histochem. Cytochem. 30 (1982) 305.

[11] J. Manston and E. Katchburian, J. Microsc. 134 (1984) 177.

[12] H. Shuman and A.P. Somlyo, Ultramicroscopy 21 (1987) 23.

[13] R.D. Leapman et al., Ultramicroscopy 49 (1993) 225.

[14] We gratefully acknowledge specimen preparation by C.-E. Hsieh and K. Buttle of Wadsworth, and advice on microanalysis from R. Leapman and B. Andrews of NIH. Supported by NIH NCRR Biomedical Research Technology Program Grant RR01219.
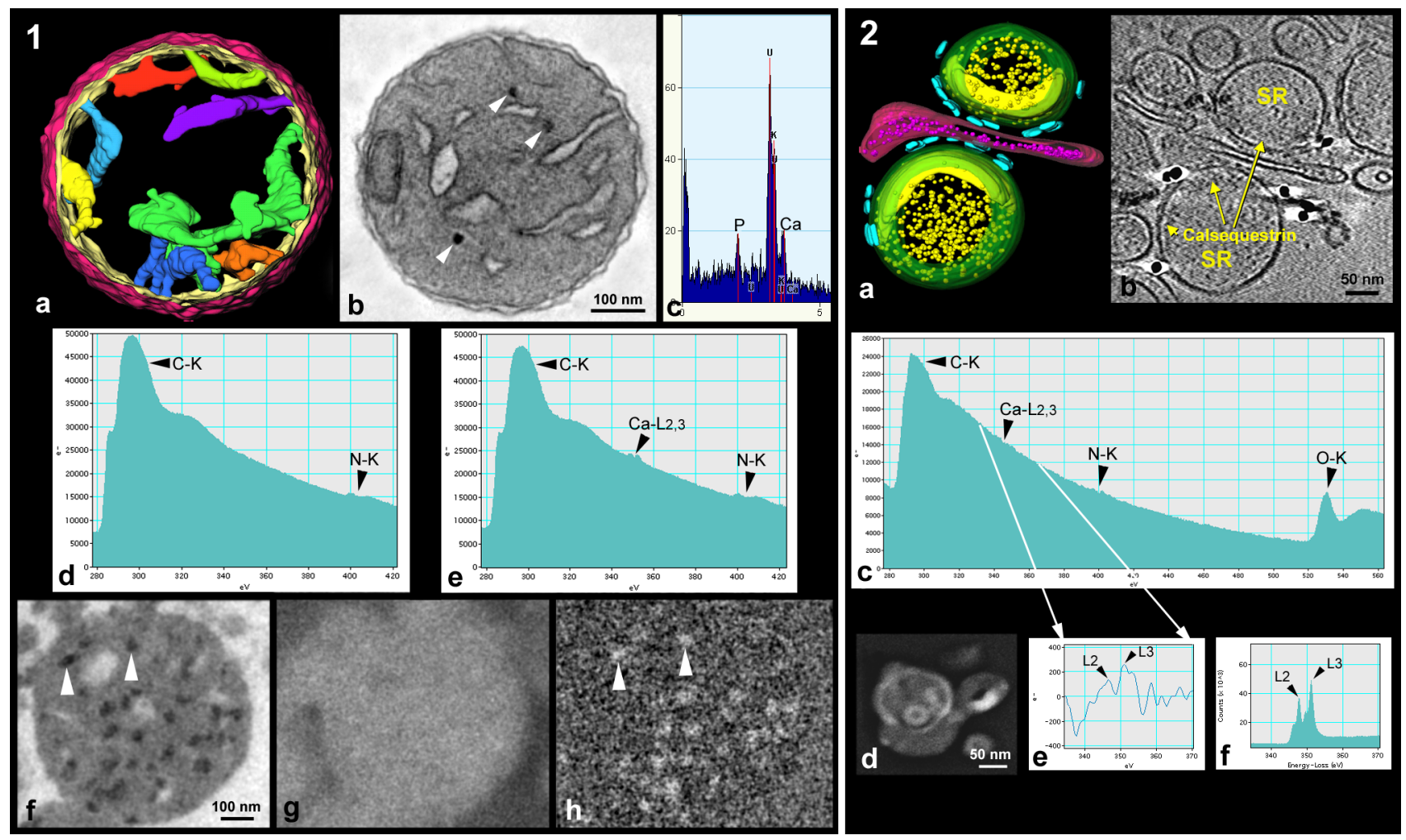

FIG. 1. Surface model (a) and tomographic slice (b) of plastic-embedded, isolated rat-liver mitochondrion; matrix granules at arrows. Using anhydrous processing [11] of similar material, as shown below in (f), X-ray EDS (c) shows Ca in granules. Typical raw EELS spectra show no $\mathrm{Ca}$ edges from matrix (d) but a clear edge at each granule (e). Unfiltered image (f), Ca pre-edge image (g) and EFTEM calcium map (h) of same specimen used for (c-e); granules at arrows.

FIG. 2. Surface model (a) and tomographic slice (b) of frozen-hydrated isolated triad junction. $\mathrm{Ca}^{++}$ should be located in SR vesicles. Raw EELS spectrum (c) shows very weak $\mathrm{Ca}$ and $\mathrm{N}$ edges from freeze-dried vesicle as shown in darkfield STEM in (d), but after first-difference processing $[12,13]$ $\mathrm{Ca} \mathrm{L}_{23}$ edges (e) that match EELS Atlas spectrum (f) in energy loss can be seen. Analysis at $400 \mathrm{kV}$ with JEM-4000FX, Gatan GIF 2002, and Emispec ES Vision. 